\section{Rapid-Onset Obesity Due to Impulsive Food- Seeking Behavior in a Puerto Rican Child With CHRNA7 15q13.3 Microdeletion}

\author{
Brian L. Jiménez ${ }^{1}$, Simón Carlo ${ }^{2}$, Wilfredo De Jesús Rojas 3, 4, 5 \\ 1. Pediatrics, Ponce Health Sciences University - School of Medicine, Ponce, PRI 2. Genetics, Ponce Health Sciences \\ University - School of Medicine, Ponce, PRI 3. Pediatric Pulmonology, University of Puerto Rico - Medical Sciences \\ Campus, San Juan, PRI 4. Pediatric Pulmonology, Ponce Health Sciences University - School of Medicine, Ponce, PRI 5. \\ Pediatric Pulmonology, San Juan Bautista School of Medicine, Caguas, PRI
}

Corresponding author: Wilfredo De Jesús Rojas, w.dejesusmd@gmail.com
Review began 03/07/2021 Review ended 03/16/2021 Published 03/20/2021

๑) Copyright 2021 Jiménez et al. This is an open access article distributed under the terms of the Creative Commons Attribution License CC-BY 4.0., which permits unrestricted use, distribution, and reproduction in any medium, provided the original author and source are credited.

\begin{abstract}
A microdeletion in the 15q13.3 locus is an exceedingly rare condition affecting the CHRNA7 gene. There have been 11 pediatric cases of this mutation reported worldwide. Clinical characteristics of the 15q13.3 microdeletion are rapid-onset obesity, hypotonia, autism, seizures, congenital cardiac defects, and neuropsychiatric disorders including impulsive hyperphagia. We describe the case of a four-year-old female with CHRNA7 15q13.3 microdeletion presenting with morbid obesity due to impulsive food-seeking behavior. We have also conducted a literature review on 15q13.3 microdeletion and compared the clinical features with other rapid-onset obesity disorders in the pediatric population. The goal of this case report is to increase awareness concerning CHRNA7 15q13.3 microdeletion as part of the differential diagnosis of rapid-onset obesity associated with neuropsychiatric disorders in pediatrics.
\end{abstract}

Categories: Genetics, Pediatrics, Pulmonology

Keywords: chrna7, 15q13.3 deletion, hyperphagia, rapid-onset obesity, developmental delay, behavioral disorder

\section{Introduction}

The CHRNA7 gene is responsible for encoding the $\alpha 7$ nicotinic acetylcholine receptor and is expressed in both the peripheral and central nervous systems [1]. CHRNA7 copy number variants have been linked to multiple cognitive and behavioral disorders, including attention deficit hyperactivity disorder (ADHD), autism spectrum disorder (ASD), epilepsy, schizophrenia, and bipolar disorder [1]. Deletions along the CHRNA7 gene, particularly 15q13.3 deletions, have been associated with severe clinical manifestations [2].

The predominant findings associated with $15 q 13.3$ microdeletions were related to neuropsychiatric conditions, primarily developmental delay and intellectual disability. Less common findings are congenital malformations, with the most common being congenital heart disease [3]. We describe the case of a pediatric patient with CHRNA7 15q13.3 microdeletion with morbid obesity of rapid-onset due to impulsive foodseeking behavior. This article was previously presented as a poster at the Puerto Rico Pediatric Society: 68th Annual Congress on February 20, 2021.

\section{Case Presentation}

The case of a four-year-old Puerto Rican female with a history of speech and motor delay, impulsive foodseeking behavior, and rapid-onset obesity since the age of two. As per the caretaker report, abnormal eating behaviors included: stealing food from others in a restaurant, eating uncooked food from the pantry and fridge, and ingesting food remnants from the garbage can. On physical examination, the patient was noted to have congenital ptosis of the right eyelid, broad nasal bridge, and velvety discoloration around her neck (Figure 1). Marked morbid obesity was also present on physical examination with a BMI above the 99.9th percentile for age and sex (Figure 2). Genetic evaluation and sleep studies ruled out other disorders associated with rapid-onset obesity including Prader-Willi syndrome and Rapid-onset Obesity with Hypothalamic Dysregulation, Hypoventilation, and Autonomic Dysregulation (ROHHAD) syndrome. Chromosomal microarray revealed a microdeletion of $443 \mathrm{~kb}$ at the $15 \mathrm{q} 13.3$ locus which explains the etiology of her phenotype and impulsive behavior. A multidisciplinary care approach was initiated for weight control and management of her additional comorbidities. Familial genetic studies were not completed in this case. 


\section{Cureus}

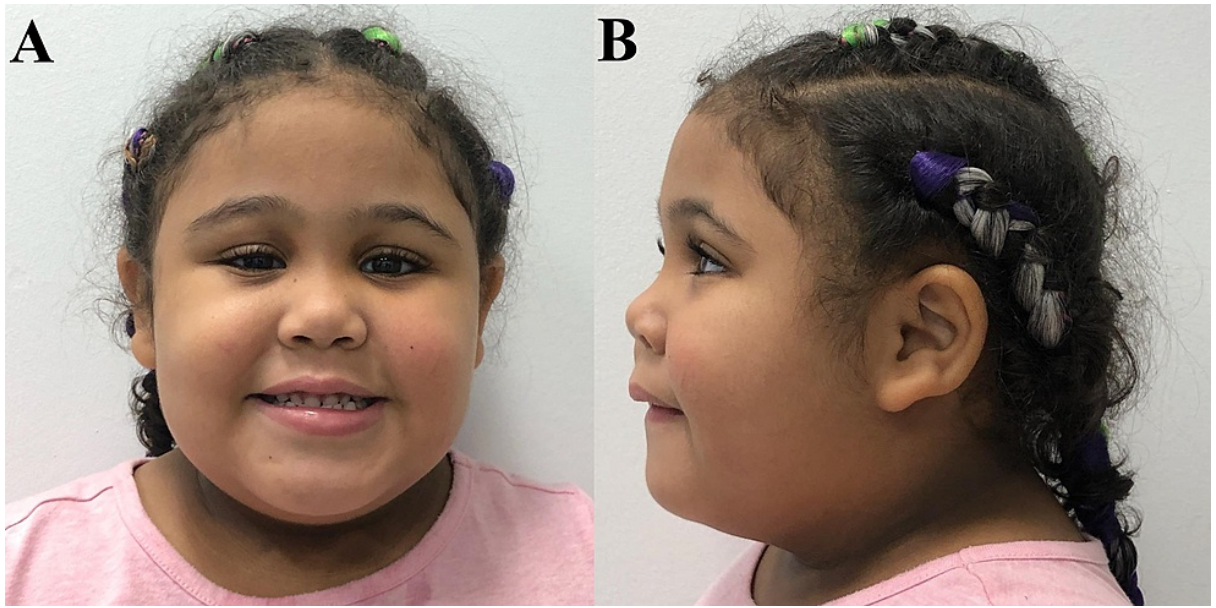

FIGURE 1: Facial features in our Puerto Rican CHRNA7 15q13.3 microdeletion case. (A): Anterior view of patient's face notable for congenital ptosis of the right eyelid. (B): Lateral view displays broad nasal bridge and velvety discoloration around her neck.

2 to 20 years: Girls

r-age percentiles
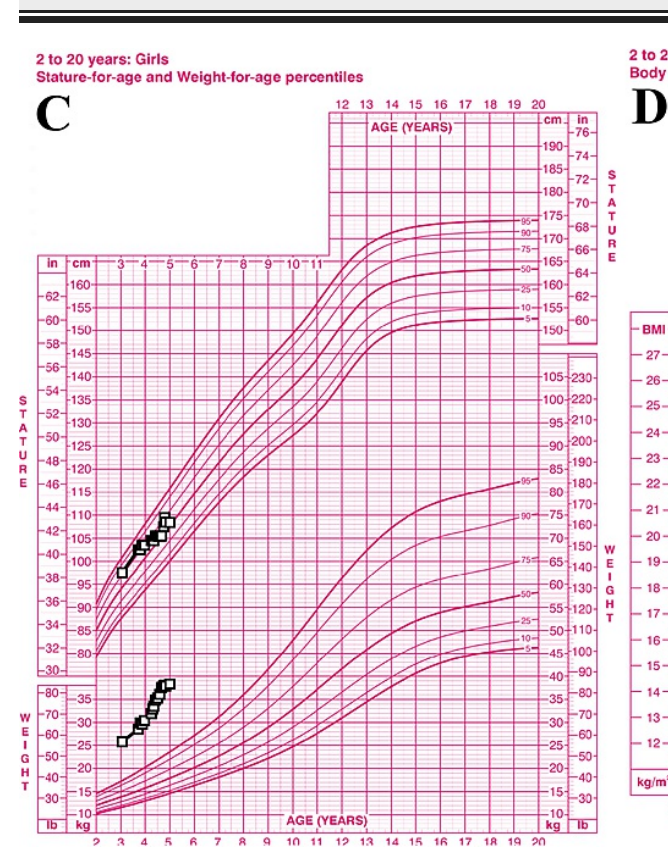

$\mathbf{C}$

FIGURE 2: Graphical representation of the patient's growth. (C):

Superior data points represent the patient's height, while inferior points represent her weight. (D): Graph of same patient's BMI, which is well above the 99.9th percentile for age and sex.

\section{Discussion}

The 15 q13.3 microdeletion syndrome is an exceedingly rare condition with a varied clinical presentation and few reported cases, with only 11 patients currently described in the literature. The various modes of regulation, multiple functions, and wide expression of CHRNA7 create significant difficulty when studying this gene [1]. The multiple neuropsychiatric conditions presenting as a result of this mutation can be difficult to distinguish from other clinical syndromes [2]. For this reason, it is especially important to differentiate the signs and symptoms from some of the syndromes that most closely resemble 15q13.3 microdeletion, namely Prader-Willi and ROHHAD syndrome (Table 1). Some of the associated diagnoses include ADHD, ASD, intellectual disability, epilepsy, schizophrenia, and bipolar disorder [3]. Neurological evaluation is also likely to reveal vision impairment, hypotonia, and abnormal electroencephalogram (EEG) findings [3]. 


\section{Cureus}

\begin{tabular}{|c|c|c|c|}
\hline Clinical Feature & 15q13.3 Microdeletion & Prader-Willi Syndrome & ROHHAD \\
\hline PW-like features (obesity) & $\mathrm{X}$ & $\mathrm{X}$ & $\mathrm{X}$ \\
\hline Hyperphagia & $\mathrm{x}$ & $\mathrm{x}$ & $\mathrm{x}$ \\
\hline Mild dysmorphic features & $X$ & $x$ & \\
\hline Intellectual disability & $X$ & $\mathrm{X}$ & $\mathrm{x}$ \\
\hline Developmental delay & $\mathrm{x}$ & $\mathrm{x}$ & $\mathrm{x}$ \\
\hline Speech/Language impairment & $X$ & $x$ & $X$ \\
\hline Autism spectrum disorder & $x$ & $x$ & $\mathrm{x}$ \\
\hline ADHD & $x$ & $x$ & $\mathrm{x}$ \\
\hline Schizophrenia & $\mathrm{x}$ & $x$ & \\
\hline Multiple congenital abnormalities & $X$ & & \\
\hline Congenital heart disease & $x$ & & \\
\hline Hypotonia & $\mathrm{x}$ & $\mathrm{x}$ & \\
\hline Seizures & $x$ & $x$ & $\mathrm{X}$ \\
\hline EEG abnormalities & $x$ & & \\
\hline Visual impairment & $\mathrm{x}$ & & \\
\hline Genital hypoplasia & & $x$ & \\
\hline Growth hormone deficiency & & $x$ & $x$ \\
\hline Hypothyroidism & & & $\mathrm{x}$ \\
\hline Hyperprolactinemia & & & $\mathrm{x}$ \\
\hline Hypoventilation & & & $x$ \\
\hline
\end{tabular}

TABLE 1: Comparison of clinical features in our case with others previously reported in the literature.

ROHHAD: Rapid-onset Obesity with Hypothalamic Dysfunction, Hypoventilation, and Autonomic Dysregulation; PW-like: Prader-Willi-like; ADHD: Attention-deficit hyperactivity disorder; EEG: Electroencephalogram.

At present, there are no specific guidelines or algorithms in place to standardize the treatment of this condition. The current strategy is to establish a multidisciplinary team of specialists to surveil, treat, and establish continuity of care [3]. Once the diagnosis has been confirmed, referral to other specialists should be based on the previously reported complications. Nutritionist consultation is essential in cases with foodseeking behavior and rapid-onset obesity, though these patients will benefit the most from strict supervision and barriers preventing access to food. Neurology should evaluate and perform EEG, if indicated, given the increased incidence of abnormal findings in these patients [4]. Though congenital anomalies are rare, congenital heart disease is among the most common and warrants an evaluation by cardiology with an echocardiogram (ECHO). Endocrinology referral for disorders of growth and metabolism is also pertinent. Ophthalmology evaluation for detection and treatment of vision impairment should be considered. A referral to a geneticist requires consideration as patients will benefit from genetic counseling. Complete pulmonary function testing and polysomnography evaluating for respiratory and sleep disorders associated with obesity, such as obstructive sleep apnea or obesity-hypoventilation syndrome, are highly recommended. A speech-language pathologist should assess for complications related to speech and developmental delay, especially given that ASD is noted to have high penetrance in 15q13.3 microdeletion patients [5]. Recommended subspecialists and screening are further detailed in Table 2. 


\section{Cureus}

declared that they have no financial relationships at present or within the previous three years with any organizations that might have an interest in the submitted work. Other relationships: All authors have declared that there are no other relationships or activities that could appear to have influenced the submitted work.

\section{Acknowledgements}

Hispanic Center of Excellence, University of Puerto Rico School of Medicine, Grant Number: D34HP24463, U.S. Department of Health and Human Services, Health Resources and Services Administration, Bureau of Health Workforce

\section{References}

1. Sinkus ML, Graw S, Freedman R, Ross RG, Lester HA, Leonard S: The human CHRNA7 and CHRFAM7A genes: a review of the genetics, regulation, and function. Neuropharmacology. 2015, 96:274-288. 10.1016/j.neuropharm.2015.02.006

2. Gillentine MA, Schaaf CP: The human clinical phenotypes of altered CHRNA7 copy number . Biochem Pharmacol. 2015, 97:352-362. 10.1016/j.bcp.2015.06.012

3. Lowther C, Costain G, Stavropoulos D, et al.: Delineating the 15q13.3 microdeletion phenotype: a case series and comprehensive review of the literature. Genet Med. 2014, 17:149-157. 10.1038/gim.2014.83

4. Simon J, Stoll K, Fick R, Mott J, Lawson-Yuen A: Homozygous 15q13.3 microdeletion in a child with hypotonia and impaired vision: a new report and review of the literature. Clin Case Rep. 2019, 7:2311-2315. 10.1002/ccr3.2403

5. Ziats MN, Goin-Kochel RP, Berry LN, et al.: The complex behavioral phenotype of 15q13.3 microdeletion syndrome. Genet Med. 2016, 18:1111-1118. 10.1038/gim.2016.9

6. Le Pichon JB, Yu S, Kibiryeva N, Graf WD, Bittel DC: Genome-wide gene expression in a patient with 15q13.3 homozygous microdeletion syndrome. Eur J Hum Genet. 2013, 21:1093-1099. 10.1038/ejhg.2013.1 